\title{
Electronic Target for Ground Based SAR Displacement Measurements
}

\author{
Mihai-Liviu Tudose ${ }^{1}$, Andrei Anghel ${ }^{2}$, Remus Cacoveanu ${ }^{2}$, Mihai Datcu ${ }^{1,3}$ \\ ${ }^{1}$ Center of Earth Observation and Space Technology, Bucharest, Romania \\ ${ }^{2}$ Telecommunications Departament, University Politehnica of Bucharest, Romania \\ ${ }^{3}$ German Aerospace Center, Remote Sensing Technology Institute, Oberpfaffenhofen, Germany \\ Contact author email: mihai_tudose89@yahoo.com
}

\begin{abstract}
This paper presents an electronic target designed for synthetic aperture radar systems. The electronic target features distinct antennas for transmitting and receiving. The electronic target basically receives a signal, down-converts it to baseband, applies a controllable time delay on the complex envelope, up-converts the delayed signal and transmits it towards the receiver of the SAR system. Since the delay is controllable, it can be used as artificial target with adjustable range. Even if the electronic target is placed in a cluttered area, its radar reflection can be moved away from its physical position. The performances of the electronic target when used for displacement measurements are evaluated.
\end{abstract}

Keywords-electronic target; SAR, radar, delay, displacement

\section{INTRODUCTION}

Ground based synthetic aperture radar (GB-SAR) is used in the fields of infrastructure elements monitoring [1] [2], terrain deformation (landslide and avalanche) detection [3], early warning systems, and for detection of structural changes in buildings. GB-SAR can be used for displacement measurements on a real target and also on an electronic target, in both monostatic and bistatic configurations.

The described instrument is used for accurate displacement measurements of remote targets, in monostatic configuration. In the present background, an electronic target (or transponder, as named in [4]) is useful for testing and calibrating SAR interferometric systems. Electronic targets have also been successfully used in the past as control points for height determination [5], named polarimetric active radar calibrators.

The electronic target (transmitter-responder) presented in this paper practically emulates a target at a configurable range (using a controlled delay) in the same azimuth position as its physical location.

The procedure helps at separating the echo provided by the electronic target from clutter (echoes from real targets arriving from the same direction). Therefore, the emulated target can be placed in a clutter-free area, by adjusting the delay. It is useful when placed on a real target (building) that is supposed to be monitored or as a radar system calibrator.

The proposed electronic target downconverts the radiofrequency signal from the receive antenna and memorizes the baseband samples. It applies a programmable time delay, then upconverts the signal and transmits it using another antenna. The input stage amplification, the output power of the transmitted signal and the carrier frequency are all configurable. Other electronic targets like the "ECR-C" are known to produce a fixed delay without any downconversion/upconversion, actually adding the delay directly in the RF domain [6]. The mentioned product is named "active transponder", and has the purpose to receive the weak signal from radar satellite, to amplify it and transmit it back to the satellite. It does not allow for adjustable delay, nor carrier frequency. Both the receive and transmit antennas are oriented in the same direction, allowing the usage in monostatic systems only.

The electronic target described in this paper features two antennas, which can be oriented in any direction independently, allowing for a higher degree of flexibility, suitable for bistatic SAR geometry.

\section{DESCRIPTION OF THE ELECTRONIC TARGET}

\section{A. Hardware description}

The electronic target is practically an FPGA implementation on the "USRP-2954R" platform. The "USRP" is the acronym for "Universal Software Radio Peripheral", a platform with dual radio transceivers, which offers a broad range of $\mathrm{TX} / \mathrm{RX}$ frequencies and a large instantaneous bandwidth $(160 \mathrm{MHz})$.

The present electronic target works at a carrier frequency of $5.755 \mathrm{GHz}$ and uses both the available RF transceiver daughterboards. From the first daughterboard, only the RX signal chain is used and the second one uses the TX signal chain only, in order to minimize unwanted leakage. The local oscillators from both daughterboards are phase locked to a single reference, but constant phase shifts between them were observed in practice.

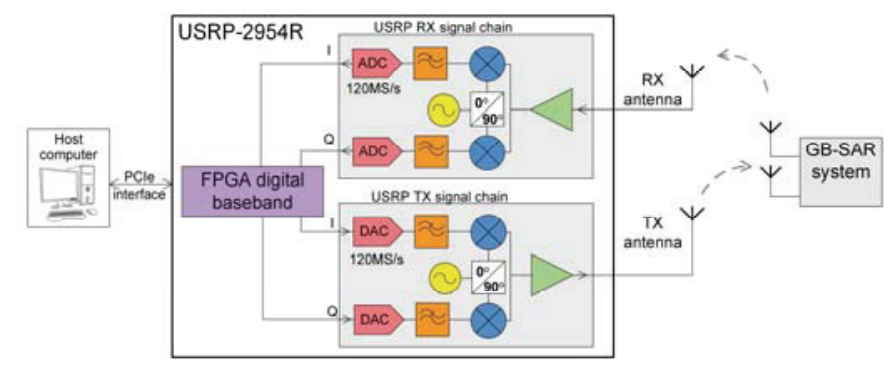

Fig. 1. Block diagram of the electronic target system 
A diagram for the entire electronic target and transponder system is presented in figure 1. The platform is controlled by a host computer using a PCIe interface. Both of the transceivers deliver/receive baseband samples to/from the FPGA digital baseband section.

\section{B. FPGA baseband implementation}

The received baseband sample is written to a memory each sampling period. The address at which the data is written is offered by a counter, which increments each sampling period. Practically, it is a circular memory. A simplified block diagram is presented in figure 2.

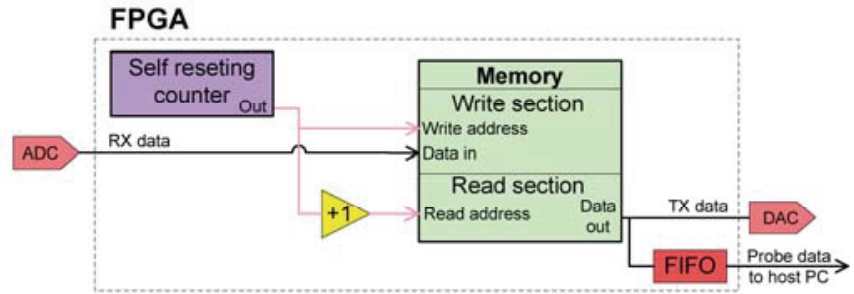

Fig. 2. Simplified block diagram of the FPGA baseband section

Data is read at the next address than the one at which the write process currently takes place. The delay introduced is equal to the memory length times the sampling period. Therefore, the delay can be adjusted by modifying the memory size.

Additional unwanted delays add up to the configured value, due to the analog front-ends sections of the transmitter and receiver group delays. The delays were estimated at a total value around $0.7 \mu \mathrm{s}$.

\section{Calibration procedure}

The "USRP-2954R" features an automated IQ imbalance correction procedure developed by the manufacturer, which is executed at the user's demand, every time a new daughterboard is installed. The correction coefficients are stored in the memory and are particular to each daughterboard.

Furthermore, in order to reduce the carrier power level, another calibration procedure was developed by the authors. Baseband ADC I and Q values on the RX part are summed with fixed positive or negative coefficients. The coefficients were determined empirically, by measuring the carrier level at the specific frequency of $5.755 \mathrm{GHz}$. This procedure allowed for a reduction in the carrier power level of at least $40 \mathrm{~dB}$.

\section{Electronic TARget RANGE COMPRESSION}

\section{A. Range compressed signal expression}

The electronic target is used with a ground-based monostatic SAR system composed of a transmitter that can move on rail to form an azimuth aperture and a fixed receiver. The ground based transmitter generates a chirp signal written as:

$$
s_{T X}(t)=\exp \left[j\left(2 \pi f_{0} t+\frac{\alpha t^{2}}{2}+\varphi_{0}\right)\right]
$$

where $\alpha$ is the chirp angular rate, $f_{o}$ is the carrier frequency and $\varphi_{0}$ is the initial phase.
The received signal on the electronic target side is a delayed version of the transmitted signal, delayed by $t_{r}$, which is proportional to the transmitter-electronic target distance (2):

$$
\mathrm{S}_{\mathrm{RX} \text {-el_target }}=s_{T X}\left(t-t_{r}\right)
$$

The electronic target downconverts the signal to baseband using homodyne architecture. It uses a local oscillator which generates a carrier and it's nominally $90^{\circ}$ phase shifted version. The receive path $\mathrm{LO}$ suffers from a random frequency shift $\Delta \mathrm{f}_{\mathrm{LO}}$ and a certain initial phase $\varphi_{\mathrm{LO} \mathrm{RX}}(3)$.

$$
s_{L O_{-} R X}(t)=\exp \left[j\left(2 \pi f_{0} t+2 \pi \Delta f_{L O} t+\varphi_{L O_{-} R X}\right)\right]
$$

This signal is mixed with the received signal, and the baseband signal (4) is formed.

$$
\begin{aligned}
& s_{B B}(t)=s_{R X-e l \_t \text { arget }}(t)>s_{L O_{-} R X} *(t)= \\
& =\exp \left[j\left(-2 \pi \Delta f_{L O} t+\frac{\alpha\left(t-t_{r}\right)^{2}}{2}+\varphi_{0}\right)\right] \times \exp \left[j\left(-\varphi_{L O_{-} R X}-2 \pi f_{0} t_{r}\right)\right]
\end{aligned}
$$

Afterwards, the baseband signal is delayed with a predefined time $t_{\text {del }}$ using the structure presented in the previous paragraph.

$$
\begin{aligned}
& s_{B B_{-} \text {del }}(t)=\exp \left[j\left(-2 \pi \Delta f_{L O}\left(t-t_{d e l}\right)\right]>\right. \\
& \times \exp \left[j\left(\frac{\alpha\left(t-t_{r}-t_{d e l}\right)^{2}}{2}+\varphi_{0}-\varphi_{L O_{-} R X}-2 \pi f_{0} t_{r}\right)\right]
\end{aligned}
$$

The delayed signal is denoted $\mathrm{S}_{\mathrm{BB}-\mathrm{del}}(\mathrm{t})$, which is upconverted using the transmit LO signal (3) on the transmit side of the electronic target. It was experimentally observed that a phase shift exists between the transmit path LO and the receive path LO. Therefore, the phases will be accounted separately, as $\varphi_{\text {LO_RX }}$ and $\varphi_{\text {LO_TX. The signal is then radiated }}$ using the TX antenna.

$$
\begin{aligned}
& s_{T X-x p o n d e r}(t)=s_{\text {bbdel }}(t) \times s_{L O_{-} T X}(t)= \\
& =\exp \left[j\left(2 \pi f_{0}\left(t-t_{r}\right)+\frac{\alpha\left(t-t_{r}-t_{d e l}\right)^{2}}{2}\right] \times\right. \\
& \times \exp \left[j\left(2 \pi \Delta f_{L O} t_{d e l}+\varphi_{0}-\varphi_{L O_{-} R X}+\varphi_{L O_{-} T X}\right)\right]
\end{aligned}
$$

The transmitted signal (6) is received by the ground radar receiver with a supplementary delay $\mathrm{t}_{\mathrm{r}}$ :

$$
\begin{aligned}
& s_{R X_{\text {rradar }}}(t)=\exp \left[j\left(2 \pi f_{0}\left(t-2 t_{r}\right)\right] \times\right. \\
& \times \exp \left[j\left(\frac{\alpha\left(t-2 t_{r}-t_{d e l}\right)^{2}}{2}+\varphi_{0}\right)\right] \times \\
& \times \exp \left[j\left(2 \pi \Delta f_{L O_{d e l}} t_{\text {d }}-\varphi_{L O_{-} R X}+\varphi_{L O_{-} T X}\right)\right]
\end{aligned}
$$

Assuming that both the transmitter and the receiver of the ground-based radar system are GPS disciplined, the received signal is down converted to baseband with the same carrier frequency. It can be shown that if the range compression is performed using a delayed version of the transmitted signal (which is received directly by the receiver on a separate direct path), the range compressed signal in baseband is [7]: 


$$
\begin{aligned}
& s_{r c_{-} b b}(t)=p s f\left[t-\left(2 t_{r}+t_{d e l}\right)\right]>\exp \left[-j 2 \pi f_{0}\left(2 t_{r}\right)\right]> \\
& \times \exp \left[j 2 \pi \Delta f_{L O} t_{d e l}\right] \times \exp \left[j\left(-\varphi_{L O_{-} R X}+\varphi_{L O_{-} T X}\right)\right]
\end{aligned}
$$

where $p s f()$ is the range point spread function (the autocorrelation function of the complex envelope $s(t))$.

The range compressed signal of the electronic target contains 4 important terms: the point spread function, the phase history (carrier) term and two phase offsets: one corresponding with the electronic target's frequency shift and another one determined by the phase difference between the local oscillators used for transmit and receive [7]. Note that the $\operatorname{psf}()$ function that corresponds to the signal's envelope has a different delay than the phase history due to the artificial delay of the electronic target, which is applied in baseband and does not contribute to the phase term.

In order to perform an azimuth focusing of the electronic target, in the focusing algorithm the phase history of the azimuth matched filter (that is not influenced by $t_{d e l}$ ) has to be artificially shifted to the position of the $p s f()$ in the range profile. Otherwise, since the target's artificial delay is comparable or larger than the physical delays (corresponding to the distances between the transmitter/receiver and the target), the artificial target can be highly defocused.

The final term yields a spurious constant phase component which is proportional to $\Delta \mathrm{f}_{\mathrm{LO}}$. A numerical estimation of the displacement $\delta \mathrm{r}$ caused by a $\Delta \mathrm{f}_{\mathrm{LO}}$ shift is approximately $100 \mu \mathrm{m}$ per $29 \mathrm{KHz}$ frequency shift at $5.755 \mathrm{GHz}$ carrier frequency and 133ns electronic target delay. Therefore, the $\Delta \mathrm{f}_{\mathrm{LO}}$ shift must be below tens of kilohertz from one acquisition to another in order to use the electronic target for interferometric purposes.

\section{B. SAR focusing result}

The SAR image focusing test using the "backprojection" algorithm was performed on a scene containing the electronic target. The GB-SAR operates in the $\mathrm{C}$ band, at a frequency of $5.755 \mathrm{GHz}$. The transmitted chirp bandwidth is $50 \mathrm{MHz}$ and lasts for $50 \mu \mathrm{s}$. The radar transmitter is moving on a $2 \mathrm{~m}$ length linear rail and transmits an impulse every $1 \mathrm{~cm}$. Therefore, there are 200 pulses available for focusing. The radar system is placed on the rooftop of the "Rectorat" building of "UPB", while the electronic target's antennas are placed on the facade of the "Leu A" building, at a line-of-sight distance of approximately $680 \mathrm{~m}$.

The focused image using "backprojection" algorithm can be observed in figure 3, after the phase history correction was performed [8].

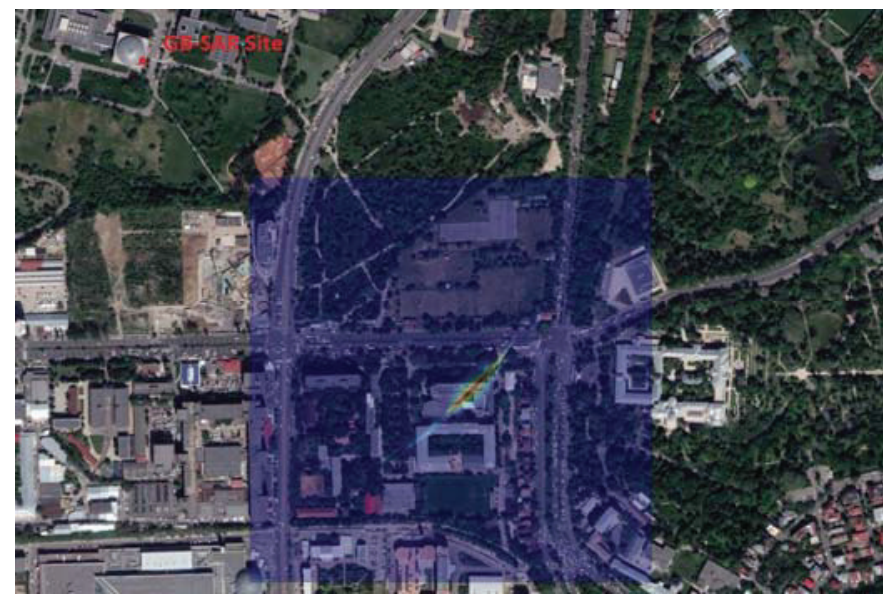

Fig. 3. SAR focused image using "backprojection". The GB-SAR site and the electronic target strong reflection on the "Leu A" building are clearly visible.

\section{DisPlacement MEASUREMENT IN-Field RESUlts}

In order to test the usability of the electronic target, displacement measurements were performed using SAR interferometry. The setup is the same one as presented in the previous chapter, with the single difference that two transmit antennas are used on the electronic target, as presented in figure 4.

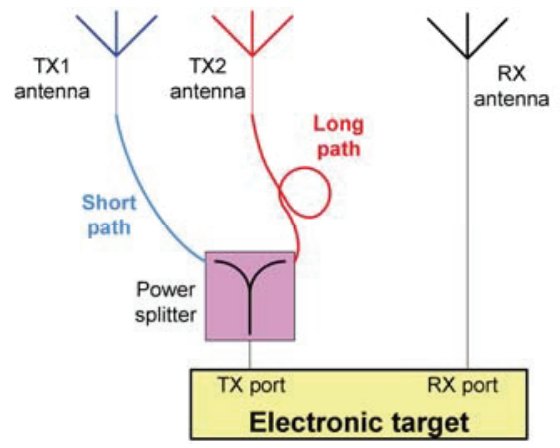

Fig. 4. Electronic target displacement measurement setup

From the two antennas, TX2 is always at a fixed position (used as phase reference), while TX1 is movable using two screws. The two antennas are connected to the transponder using different length coaxial cables because their reflections need to be clearly separated in range.

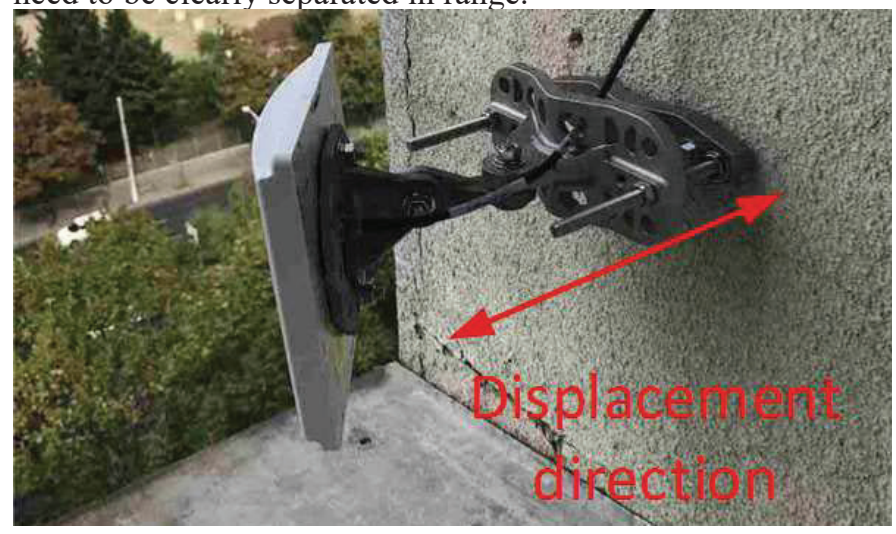

Fig. 5. Displacement direction of TX1 antenna 
A photograph of TX1 antenna can be observed in figure 5 . The displacement direction marked in the photograph is $45^{\circ}$ away from the ground-based LOS direction.

Two in-situ displacements sets were performed for TX1. The first one starts at $0 \mathrm{~mm}$, increases approximately linear to $25 \mathrm{~mm}$ and than decreases to $0 \mathrm{~mm}$. The second displacement set involves moving the TX1 antenna between $0 \mathrm{~mm}$ and $14 \mathrm{~mm}$ fixed positions at each of the acquisitions. The SNR was approximately $30 \mathrm{~dB}$.

\section{A. Data set 1}

Figure 6 shows the estimated relative displacements (between TX1 and TX2 antennas) projected in the GB line of sight for both antennas.

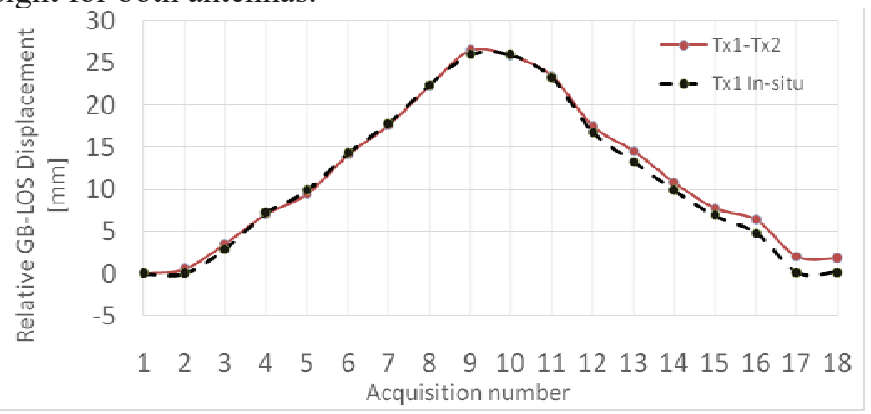

Fig. 6. Measurement displacements for TX1 movable antenna relative to the TX2 fixed antenna and the TX1 in-situ displacements - data set 1

In order to compensate for atmospheric effects, the displacements were measured between TX1 and TX2.

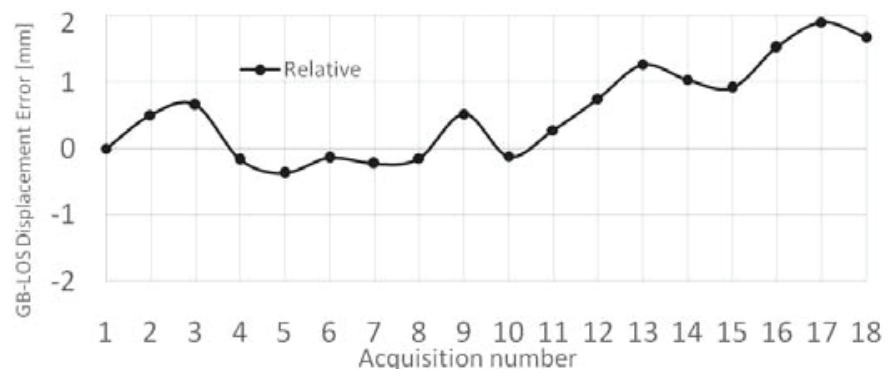

Fig. 7. Error of the TX1-TX2 displacement measurement for set 1

The errors for the TX1 relative to TX2 displacement measurements are presented in figure 7 . The displacement measurement error is always lower than $2 \mathrm{~mm}$.

\section{B. Data set 2}

For the second data set, the displacements of TX1 relative to TX2 antenna are presented in figure 8 .

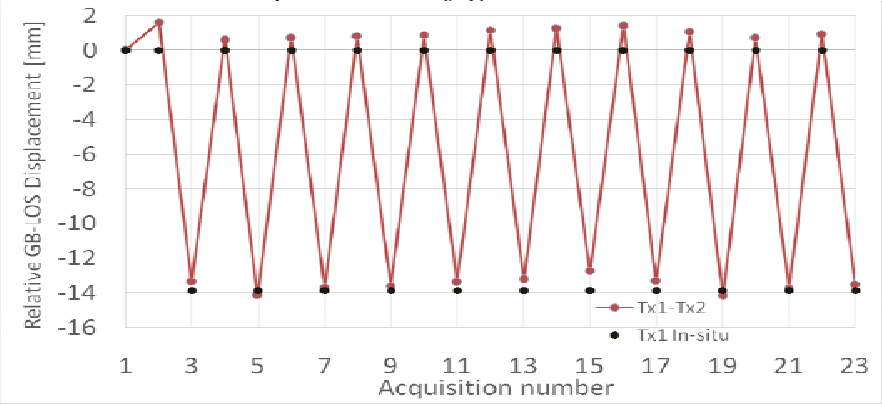

Fig. 8. Displacements of TX1 antenna relative to TX2 antenna measurements for data set 2
TX1 measured displacements are very close to the in-situ values. The error for this data set can be observed in figure 9.

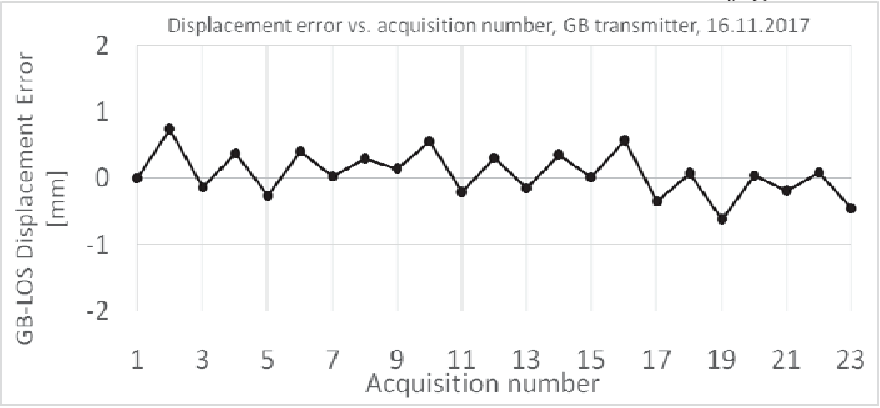

Fig. 9. Error of the displacement of TX1 relative to TX2 measurement for set 2

In this case, the error is below $1 \mathrm{~mm}$.

\section{CONCLUSIONS}

The electronic target is suited for displacement measurement, in a scene scanned by a GB-SAR. The relative displacement measurement errors are below $2 \mathrm{~mm}$ for an electronic target physically located at a line of sight distance of approximately $680 \mathrm{~m}$.

The electronic target is useful at shifting the location of the target out of a cluttered area and performing measurements at better signal to noise ratio. Therefore, in the future, the designed electronic target can be used as active radar calibrator for displacement measurements applications.

\section{ACKNOWLEDGEMENT}

This work has been partly funded by University Politehnica of Bucharest, through the "Excellence Research Grants" Program, UPB - GEX 2017, Identifier: UPBGEX2017, Ctr. No. 37/25.09.2017 (AMIRAD project).

\section{REFERENCES}

[1] A. Anghel, G. Vasile, R. Cacoveanu, C. Ioana, S. Ciochină, "Shortrange wideband FMCW radar for millimetric displacement measurements," IEEE Trans. on Geoscience and Remote Sensing, 2014, 52, (9), pp. 5633-5642.

[2] L. Cunlong, C. Weimin, L. Gang, Y. Rong, X. Hengyi, Q. Yi, “A noncontact FMCW radar sensor for displacement measurement in structural health monitoring," Sensors, 2015, 15, (4), pp. 7412-7433.

[3] O. Monserrat, M. Crosetto, G. Luzi, "A review of ground-based SAR interferometry for deformation measurement," ISPRS Journal of Photogrammetry and Remote Sensing, 2014, 93, pp. 40-48.

[4] K. Dumper, C. Buck, S.R. Day, D. King, P.E. Gomm, "Design and performance of the prototype advanced SAR calibration transponder," CEOS_SAR 2004.

[5] S. Duque, P. Lopez-Dekker, J.C. Merlano, J.J Mallorqui, "Bistatic SAR tomography: Processing and Experimental Results," IGARSS 2010

[6] ECR-C, http://www.metasensing-group.com/ecr-c, accesed on 4.11.2017

[7] A. Anghel, R. Cacoveanu, A. S. Moldovan, A. A. Popescu, M. Datcu, and F. Serban, "Simplified bistatic SAR imaging with a fixed receiver and TerraSAR-X as transmitter of opportunity - First results," IEEE International Geoscience and Remote Sensing Symposium (IGARSS), Beijing, China, Jul. 2016, pp. 2094-2097.1982.

[8] M. Tudose, A. Anghel, R. Cacoveanu, M. Datcu, "Electronic target for bistatic/monostatic SAR systems," EUSAR 2018, Aachen, Germany, in press. 